EUROPEAN ORGANIZATION FOR NUCLEAR RESEARCH

European Laboratory for Particle Physics

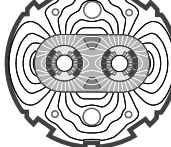

Large Hadron Collider Project

LHC Project Report 120

\title{
SUMMARY OF THE SINGLE-BEAM COLLECTIVE EFFECTS IN THE LHC
}

Berg, J.S.; Bruening, O.; Caspers, F.; D'yachkov, M.*; Morvillo, M.; Ruggiero, F.

\begin{abstract}
Single beam collective effects can limit the performance of the LHC and, together with parasitic losses, impose constraints on the design of the components of the LHC vacuum system. Because of the large number of bunches in the LHC, coherent losses and multibunch instability rise times generated by the impedance items can be rather large. For example, the vacuum chambers of the main experiments can lead to large parasitic losses and a continuous feedback from the impedance calculations during their design process is desirable. We first summarise the current status of the impedance model for the LHC and estimate rise times and threshold currents for different instabilities. In a second step, we discuss possibilities of controlling these instabilities, using feedback systems and Landau damping.
\end{abstract}

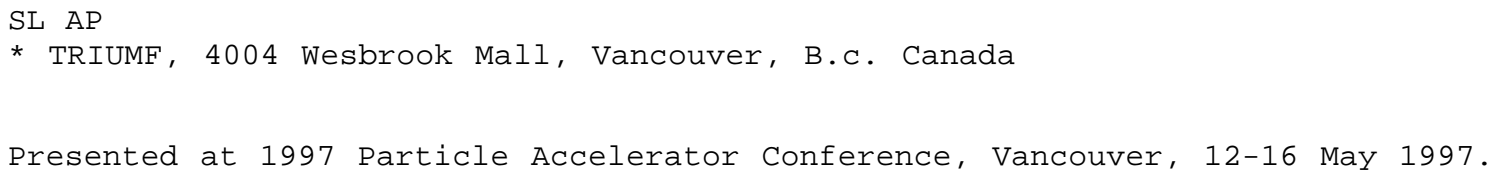

Geneva, 11 juin 1997 


\title{
Summary of the Single-Beam Collective Effects in the LHC
}

\author{
F. Ruggiero, J.S. Berg, O. Brüning, F. Caspers, M. Morvillo, CERN, Geneva, Switzerland \\ and M. D'Yachkov, TRIUMF, 4004 Wesbrook Mall, Vancouver, B.C., Canada V6T 2A3
}

\section{Abstract}

Single beam collective effects can limit the performance of the LHC and, together with parasitic losses, impose constraints on the design of the components of the LHC vacuum system. Because of the large number of bunches in the LHC, coherent losses and multibunch instability rise times generated by the impedance items can be rather large. For example, the vacuum chambers of the main experiments can lead to large parasitic losses and a continuous feedback from the impedance calculations during their design process is desirable. We first summarise the current status of the impedance model for the LHC and estimate rise times and threshold currents for different instabilities. In a second step, we discuss possibilities of controlling these instabilities, using feedback systems and Landau damping.

\section{INTRODUCTION}

The performance of the LHC will depend on the beam intensity, which may be limited by collective effects unless the impedance of the different vacuum chamber discontinuities is kept below certain limits. At the design stage of the machine, a fast and continuous feedback from impedance calculations or measurements to hardware builders is thus necessary. An impedance database program (ZBASE [1]) has therefore been setup and already successfully applied to estimate the LEP impedance budget: it helps keeping track of all the known impedance sources, of the corresponding betatron functions and of the input files used by different programs, such as ABCI or MAFIA, to compute high order modes and loss factors for several bunch lengths. In the near future, a further interface is foreseen to existing beam dynamics codes for detailed calculations of multibunch transverse mode-coupling and microwave instability thresholds both in the LHC and SPS.

In Sec. 2 and 3, we review parasitic losses and impedance estimates recently obtained for several LHC components, mentioning also some work in progress. We then present in Sec. 4 the results of the program VLASOV [2] for calculations of coherent mode frequencies including multibunch mode coupling. Finally we discuss possibilities of controlling potentially unstable modes using feedback systems and Landau damping [3]. The ongoing activity concerning collective effects in the LHC, including recent results on space charge, Laslett coefficients and the electron cloud instability, is documented on the world wide web at the address http://wwwslap.cern.ch/collective/.

\section{PARASITIC LOSSES}

\subsection{Beam screen}

The LHC beam screen is cooled at a temperature ranging from $5^{\circ}$ to $20^{\circ} \mathrm{K}$. It is a stainless steel pipe with a $50 \mu$ copper coating that reduces its surface resistance, thus minimising at the same time transverse resistive wall instability and beam-induced wall heating. At top energy and for nominal beam parameters, the classically computed resistive wall heating of the LHC beam screen is $75 \mathrm{~mW} / \mathrm{m}$. Owing to anomalous skin effect and surface roughness, however, this value could be significantly underestimated. Since the parasitic heating scales with the square of the beam current, for the ultimate beam intensity of $850 \mathrm{~mA}$, it may become comparable to the synchrotron radiation power loss of $326 \mathrm{~mW} / \mathrm{m}$. A programme of surface resistance measurements at different temperatures, frequencies, and magnetic field intensities has therefore been launched to provide a realistic heating budget for the LHC cryogenic system and to optimise the fabrication process of the beam screen. The measurements are based on the comparison of the quality factors for even and odd TEM modes excited in a cylindrical structure with two inner conductors [4]. Preliminary results at cryogenic temperatures (without magnetic field) indicate a surface resistance about a factor two larger than previously estimated.

Resistive losses in the longitudinal welds of the beam screen, with its new racetrack geometry, are $20 \mathrm{~mW} / \mathrm{m}$ for nominal and $50 \mathrm{~mW} / \mathrm{m}$ for ultimate beam intensity. Other losses, associated with TEM coaxial modes excited by the pumping slots and travelling synchronously with the beam are presently estimated to be less then $10 \mathrm{~mW} / \mathrm{m}$ for nominal and $25 \mathrm{~mW} / \mathrm{m}$ for ultimate beam intensity. About half of this power will be deposited on the cold bore at $1.9^{\circ} \mathrm{K}$.

\subsection{Experimental vacuum chambers}

Another source of parasitic losses are the experimental beam pipes with their cavity like structures which give rise to a large number of narrow band HOM's. For a narrow band impedance, the wake fields of different bunches can overlap, leading to a coherent power loss proportional to the square of the total number of bunches $N_{b}$. If $\omega_{0}=$ $2 \pi \cdot f_{\text {rev }}$ is the angular revolution frequency, $Q_{b}$ the charge per bunch, $\tau_{n}$ the position of the $n^{t h}$ bunch, $\sigma_{z}$ the bunch length and $Z_{\|}$the longitudinal impedance, we have

$$
\begin{aligned}
P_{c o h .}= & -f_{r e v}^{2} \cdot Q_{b}^{2} \cdot \sum_{p=-\infty}^{+\infty}\left|\sum_{n=1}^{N_{b}} \exp \left(-i p \omega_{0} \cdot \tau_{n}\right)\right|^{2}(1) \\
& \times \exp \left(-\left[p \cdot \omega \sigma_{z}\right]^{2} / c^{2}\right) \cdot Z_{\|}\left(p \omega_{0}\right) .
\end{aligned}
$$




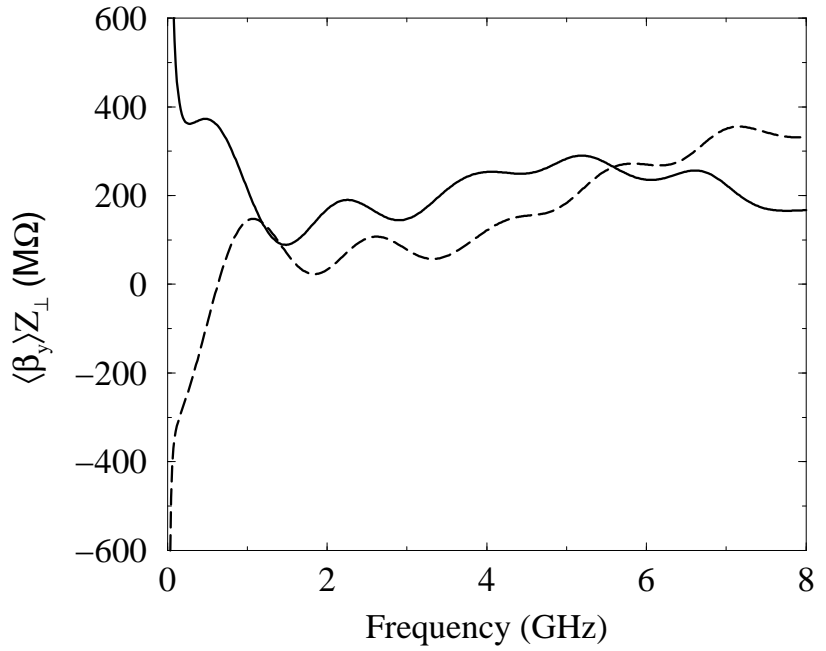

Figure 1: Vertical broad band impedance times average $\beta$ function for the LHC: real (solid line) and imaginary part (dashed line).

The LHC has 2835 bunches with a bunch length of $13 \mathrm{~cm}$ at injection and $7.5 \mathrm{~cm}$ at top energy. At the moment there are four experiments foreseen in the LHC: the two main experiments CMS and ATLAS, plus LHC-B and ALICE. For example, the current design of the CMS vacuum chamber has about 30 HOM's with Q-values larger than 30000 and with mode frequencies within the bunch spectrum for a $13 \mathrm{~cm}$ long bunch. When lying on a line of the bunch spectrum, each of these HOM's can lead to a coherent power loss of more than $1 \mathrm{~kW}$.

\section{IMPEDANCE MODEL}

A detailed description of the impedance model used for the LHC can be found in Refs. [5] and [6]. Here we discuss only recent updates in some of our impedance estimates. A plot of the broad band impedance used in the calculations is shown in Fig. 1. The narrow band impedance is the same as in [6], but we assume that the most prominent transverse mode in the longitudinal feedback cavities (the one at $516.7 \mathrm{MHz}$ ) is damped down to a $Q$-value of 1000 .

\subsection{Bellows and BPM's}

Owing to the 3D geometry of the bellows, the code MAFIA was used for wake field and impedance calculations. The results presented in a companion paper [7] indicate that the latest bellows design, with smooth $10 \mathrm{~mm}$ transitions from racetrack to round geometry and shielding provided by sprung fingers which can slide along the beam screen, has impedances smaller than those previously estimated according to a rather conservative scaling of SSC calculations and LEP measurements. For a double $1 \mathrm{~mm}$ step and 3000 bellows, the low-frequency longitudinal impedance is $Z / n=18.3 \mathrm{~m} \Omega$ and the vertical impedance is $753 \mathrm{k} \Omega / \mathrm{m}$. The transverse impedances of the bellows in the vertical and horizontal planes are not only different, but have also different signs (i.e., focusing in one plane and defocusing in the other). The effect of the gaps between fingers is found to be negligible.

A final design for the 500 beam position monitors is not yet available. Here we assume that each of them consists of four strip-lines, $10 \mathrm{~cm}$ long and $18 \mathrm{~mm}$ wide, located at a distance of $18 \mathrm{~mm}$ from the beam axis. A substantial reduction in length, compared to previous designs, is made possible by the proximity of the associated electronics, now located in the LHC tunnel.

\subsection{Kickers}

Impedance estimates are in progress for the LHC kickers, whose final design may depend on the outcome of possible impedance measurements foreseen in the near future.

The LHC abort kicker magnets have a total length $L=$ $14 \times 1.26 \mathrm{~m}$ and are located in a region where the vertical $\beta$ function is about $600 \mathrm{~m}$. They are equipped with a rectangular ceramic vacuum chamber of thickness $\Delta=4.5 \mathrm{~mm}$ and vertical aperture $b=14 \mathrm{~mm}$. The inside of the chamber has a titanium coating of thickness $d=1 \mu \mathrm{m}$, with resistivity $\rho_{\mathrm{Ti}}=7 \times 10^{-7} \Omega \mathrm{m}$, to reduce the high-frequency impedance seen by the beam and to conduct away the static charge. The vertical impedance at frequencies well below $1 \mathrm{GHz}$ can be written

$$
Z_{\perp}(\omega)=\frac{-i R}{1-i \omega / \omega_{C}}
$$

where $R=\frac{Z_{0} L}{2 \pi b^{2} \zeta} \simeq 8.5 \mathrm{M} \Omega / \mathrm{m}$ and $\omega_{C}=\frac{2 \zeta \rho_{\mathrm{Ti}}}{b d \mu_{0}} \simeq$ $2 \pi \times 8 \mathrm{MHz}$. Here $\zeta=(b+\Delta)^{2} /\left[(b+\Delta)^{2}+b^{2}\right] \simeq$ 0.636. Note that (contrary to Refs. [5] and [6]) we have now assumed perfect magnetic boundary conditions, better suited for the (H-shaped) kicker ferrite.

The LHC injection magnet system must produce a kick of $1.36 \mathrm{Tm}$ with a duration of $6.5 \mu$ s and consists of 4 units with a length of $2.17 \mathrm{~m}$ each. The kicker field rise time is $900 \mathrm{~ns}$ with a maximum ripple of 2 per mil. Ohmic losses in the (H-shaped) ferrite are substantially reduced by a round ceramic tube (with $18 \mathrm{~mm}$ inner radius and $4.5 \mathrm{~mm}$ thickness) painted in the inside with several $5 \mu \mathrm{m}$ thick copper stripes, that should carry most of the high-frequency beam-induced image currents. To avoid loop currents in the stripes due to $\dot{B}$, they are not directly connected to the external beam pipe. Instead, a capacitive contact (about $800 \mathrm{pF}$ ) is established thanks to the high dielectric constant $\epsilon \simeq 10$ of the ceramic tube [8]. The field pattern of possible resonant modes associated with the stripes (behaving like a strip-line with unmatched load) shall be investigated to assess their penetration into the ferrite, with consequent strong damping.

According to BINP calculations [9] using the code ILANS, the effect of the injection kickers on the transverse resistive wall instability at $8.5 \mathrm{kHz}$ is negligible: assuming an average vertical $\beta$-function of $70 \mathrm{~m}$, the real part of the transverse impedance times $\beta$ is $6 \mathrm{M} \Omega$, to be compared to 


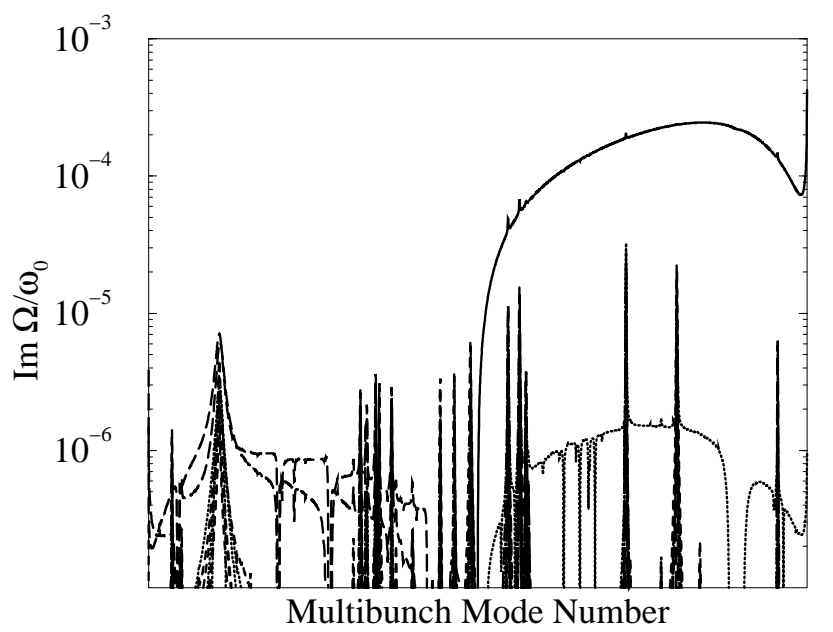

Figure 2: Vertical growth rates at injection versus multibunch mode number, for dipole (solid) and higher order modes.

about $3 \mathrm{G} \Omega$ from the resistive wall. However, the calculation is in quasi-static approximation and does not include the capacitive coupling of the stripes to the beam pipe.

As a temporary rough model for the injection kicker, we assume that the uncoated part of the ceramic chamber (about 36\%) contributes a purely inductive impedance [10] $-0.4 i \frac{Z_{0} L}{2 \pi b^{2}}\left(\frac{\alpha \epsilon-1}{\alpha \epsilon+1}+\frac{b^{2}}{(b+\Delta)^{2}}\right) \simeq-i 1.63 \mathrm{M} \Omega / \mathrm{m}$, where $\alpha=\left[(b+\Delta)^{2}-b^{2}\right] /\left[(b+\Delta)^{2}+b^{2}\right] \simeq 0.22$, while the region covered by copper stripes $(64 \%)$ contributes a transverse impedance given by Eq. (2), with $\zeta \simeq 0.61$, $R \simeq 1.7 \mathrm{M} \Omega / \mathrm{m}$ and $\omega_{C} \simeq 2 \pi \times 25.7 \mathrm{kHz}$.

\section{COHERENT INSTABILITIES}

Using the impedance model of the previous section, the vertical multibunch growth rates computed at injection for nominal beam parameters by VLASOV are shown in Fig. 2 . The plot consists of several lines, one for each type of internal-bunch motion. Each line contains one point for each multibunch mode. The computation is done for $N_{b}$ symmetrically placed bunches, with a total beam current of $674 \mathrm{~mA}$. The peak on the left side of the figure is primarily caused by two of the septum cavity higher order modes: the one at $1117.9 \mathrm{MHz}$ and the one at $516.7 \mathrm{MHz}$. The peak all the way to the right is primarily caused by the resistive-wall impedance, while the broad hump on the right is associated with the impedance of the abort kickers. Multibunch TMC occurs at about twice the nominal current.

The effect of a transverse feedback system with $1 \mathrm{MHz}$ half-bandwidth and a gain corresponding to a damping time of $10 \mathrm{~ms}$ is shown in Fig. 3, where we compare the corresponding coherent tune shifts of rigid dipole and $m=1$ head-tail modes to Landau damping stability curves for a transverse parabolic-like distribution collimated at anywhere from $3 \sigma$ to $6 \sigma$. The dashed curve is for a shift in the vertical tune with vertical amplitude of $10^{-4}$ at $1 \sigma$,

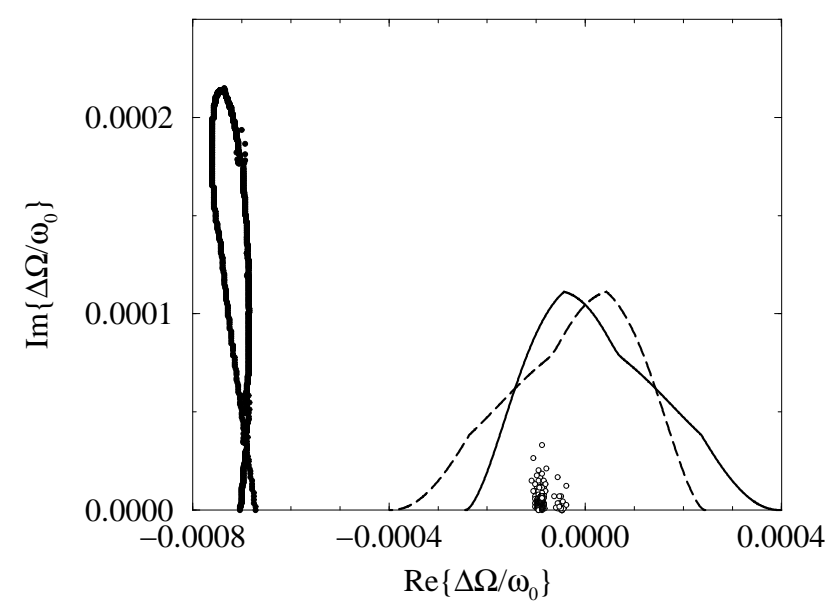

Figure 3: Vertical coherent tune shifts with $1 \mathrm{MHz}$ halfbandwidth feedback, of $m=0$ modes (filled circles, on the left) and $m=1$ modes (open circles), compared to Landau damping stability curves.

whereas the solid curve is for $-10^{-4}$. The shift in the vertical tune with horizontal amplitude has opposite sign and is $\pm 7.2 \times 10^{-5}$. We see from the figure that, although Landau damping from octupoles (and space charge) stabilises all the $m=1$ modes, some of the $m=0$ modes are unstable (essentially those driven by the impedance of the abort kickers). This is a consequence of the insufficient feedback bandwidth, which we assumed to be the same as the bandwidth of the power amplifier required to damp injection oscillations. By suitable modifications of the feedback electronics, however, it should be possible to obtain a low-power half-bandwidth of about $20 \mathrm{MHz}$ [11], thus stabilising all dipole modes at injection. Octupoles will be required at top energy to stabilise rigid dipole and higher order head-tail modes [12].

\section{REFERENCES}

[1] O. Brüning, these Proceedings.

[2] J.S. Berg, SLAC-TN-96-1 (1996).

[3] J.S. Berg and F. Ruggiero, these Proceedings, see also J.S. Berg, CERN LHC Project Report 100 (1997).

[4] F. Caspers, M. Morvillo and F. Ruggiero, these Proceedings.

[5] F. Ruggiero, Part. Accel. 50, 83-104 (1995).

[6] J.S. Berg, CERN LHC Project Report 16 (1996).

[7] M. D'yachkov and F. Ruggiero, these Proceedings.

[8] G. Schröder, private communication (January 1997).

[9] M.M. Karliner, N.V. Mityanina, D.G. Myakishev and V.P. Yakovlev, private communication (May 1996), see also Proc. EPAC Conference, Sitges, 1996 (IPP, Bristol, 1996), pp. 1247-1249.

[10] S.S. Kurennoy, in Proc. of the IEEE 1993 Part. Acc. Conf., Washington, D.C., p. 3420 (1993).

[11] W. Höfle, private communication (May 1997).

[12] J. Gareyte, J.P. Koutchouk and F. Ruggiero, CERN LHC Project Report 91/rev (1997). 